\title{
Los ODS y el transporte marítimo en México: el marco legal internacional para alcanzar las metas del ODS 3 buena salud y del ODS 13 acción por el clima
}

The SDGs and maritime transport in Mexico: the international legal framework to achieve the goals of SDG 3: Good Health and Well-Being and SDG 13 Climate Action

\author{
Pedro Alfonso Elizalde Monteagudo \\ Doctor en Derecho y la Contratación \\ Tecnológico de Monterrey - México \\ orcid 0000-0003-3425-1759 \\ pelizald@tec.mx
}

\footnotetext{
Cómo citar este artículo:

Elizalde, P. A. (2022). Los ODS y el transporte marítimo en México: el marco legal internacional para alcanzar las metas del ODS 3 buena salud y del ODS 13 acción por el clima. Revista de la Facultad de Derecho y Ciencias Políticas, 52(136), pp. 24-38. doi: https://doi.org/10.18566/rfdcp.v52n136.a02 Recibido: 11 de julio de 2020 Aprobado: 16 de marzo de 2021
} 


\section{Resumen}

No existe un Objetivo de Desarrollo Sostenible (ODS) enfocado en el transporte marítimo, sin embargo, el transporte marítimo está relacionado con varias metas de los ODS; particularmente, el transporte marítimo está íntimamente relacionado con las metas del ODS 3: Salud y Bienestar y del ODS 13: Acción por el Clima. La comunidad internacional ha generado varios instrumentos legales internacionales para coadyuvar con el éxito de las metas de los dos ODS antes mencionados. El logro de las metas generaría beneficios globales. A pesar de lo anterior, algunos países ratificantes no han podido implementar las disposiciones de tales instrumentos legales por cuestiones técnicas o económicas.

\section{Palabras clave}

Transporte marítimo; objetivos de desarrollo sostenibles; emisión de gases invernadero; azufre; zona de control de emisiones.

\section{Abstract}

There is no Sustainable Development Goal (SDG) focused on maritime transport. However, maritime transport is related to several goals and indicators contained in various Sustainable Development Goals. Particularly, maritime transport is closely related to the SDG 3: Good Health and Well-Being SDG 13: Climate Action goals. The international community has generated several international legal instruments to contribute to the achievement of the two SDGs goals mentioned above. The achievement of the goals would generate benefits at a global level.Key words: maritime transport, sustainable development goals, greenhouse gas emissions, sulfur, emission control zone.

However, some countries have not been able to implement the provisions of these legal instruments despite having ratified them due to technical or financial reasons.

\section{Keywords}

Maritime transport, sustainable development goals, greenhouse gas emissions, sulfur, emission control zone. 


\section{Listado de abreviaturas}

$\begin{array}{ll}\text { BIMCO } & \text { Consejo Marítimo Internacional y del Báltico } \\ \text { CMNUCC } & \text { Convención Marco de Naciones Unidas sobre Cambio Climático } \\ \text { CO2 } & \begin{array}{l}\text { Dióxido de Carbono } \\ \text { COP 21 }\end{array} \\ & \begin{array}{l}\text { Vigesimoprimera Conferencia de las Partes de la Convención } \\ \text { Marco de Naciones Unidas sobre Cambio Climático }\end{array} \\ \text { COVID-19 } & \text { Coronavirus 2019. } \\ \text { ECA } & \text { Zonas de Control de Emisiones } \\ \text { GEI } & \text { Gases Efecto Invernadero } \\ \text { MARPOL } & \text { Convenio Internacional para Prevenir la Contaminación por } \\ \text { NOx } & \text { Buques } \\ \text { ODS } & \text { Óxidos de Nitrógeno } \\ \text { OMI } & \text { Objetivos de Desarrollo Sostenibles } \\ \text { ONU } & \text { Organización Marítima Internacional } \\ \text { SEMARNAT } & \text { Organización de Naciones Unidas } \\ \text { SOX } & \text { Óxido de Azufre de Medio Ambiente y Recursos Naturales } \\ \text { TEU } & \text { Unidad(es) equivalente(es) a 20 Pies } \\ \text { TPP } & \text { Tratado de Asociación Transpacífico } \\ \text { UNCTAD } & \text { Conferencia de las Naciones Unidas sobre Comercio y Desarrollo }\end{array}$

\section{La Importancia del transporte marítimo en el mundo}

El transporte marítimo está intrínsecamente vinculado con el comercio internacional y el desarrollo sostenible. Al respecto, la Conferencia de las Naciones Unidas sobre Comercio y Desarrollo (UNCTAD, 2019) señala que el transporte marítimo representa el $\mathbf{8 0 \%}$ del volumen y el $70 \%$ en valor del comercio internacional. 92,295 buques participaron en el comercio marítimo internacional durante 2018. El promedio de edad de los buques es de 21 años. Además, el transporte marítimo sobrepasó los 11 mil millones de toneladas transportadas en el año antes mencionado.

En el mismo sentido, la UNCTAD (2019) estima que los puertos de todo el mundo manipularon 793,26 millones de TEU en el año 2018. La cantidad de carga contenerizada es distinta en cada uno de los continentes. La participación en el manejo de carga contenerizada por cada continente está representada de 
la siguiente manera: Asia 64\%, Europa 16\%, Norteamérica 8\%, Latinoamérica $7 \%$, África $4 \%$ y Oceanía $2 \%$.

El informe sobre transporte marítimo de la UNCTAD (2019) menciona que el comercio marítimo mundial creció durante 5 años consecutivos hasta finales del año 2017, pero, el transporte marítimo internacional presentó un crecimiento menor en los años 2018 y 2019 debido al Brexit del Reino Unido, la transición económica de China y las tensiones comerciales entre Estados Unidos y China.

Al mismo tiempo, el transporte marítimo fue afectado por la pandemia del COVID-19. Sobre el tema, la Asociación Internacional de Puertos y Terminales (2020) indica que el 39\% de los puertos presentó un decremento del 5\% al $25 \%$, y el $52 \%$ de los puertos mostró una estabilidad en el número de atraques de buques contenedores durante la semana 15 del año 2020. Por el contrario, el $45 \%$ de los puertos tuvo un decremento del 5\% al 25\%, y el $47 \%$ de los puertos experimentó una estabilidad en el número de atraques de buques contenedores durante la semana 25 del mismo año.

Por su parte, el Instituto Mexicano del Transporte (IMT, 2020) menciona que la carga comercial no petrolera tuvo una reducción del $5.1 \%$ en los puertos mexicanos durante el primer trimestre del año 2020, con respecto al mismo periodo del año 2019. En concreto, los puertos mexicanos del Pacífico despacharon 7,184,309 TEU's. A su vez, los puertos del Golfo y Caribe despacharon 4,739,470 TEU's durante enero-marzo 2020. Este número de contenedores contrasta con los 7,991,038 y 4,914,931 TEU's despachados respectivamente durante enero-marzo 2019.

\section{Las repercusiones del transporte marítimo en el medio ambiente y la salud}

La UNCTAD (2019) señala que la relación entre el transporte marítimo y el medio ambiente genera elementos de preocupación y de riesgo para el crecimiento de esta modalidad de transporte, tomando en cuenta las repercusiones que tienen las emisiones procedentes de los buques en la contaminación ambiental y el cambio climático.

En el mismo sentido, la OMI (2014) indica que las emisiones de los buques contribuyen al cambio climático y la contaminación. Tales emisiones generan 
problemas en la salud humana y lluvia ácida en las zonas costeras. En específico, la OMI (2020) señala que el óxido de nitrógeno (NOx) y óxido de azufre (SOx) tienen un efecto negativo en la salud humana. De hecho, el SOx genera problemas en el sistema respiratorio además de contribuir a la formación de lluvia ácida. Por su parte, el dióxido de carbón CO2 está considerado como un gas de efecto invernadero (GEI) procedente de los buques.

El Tercer Estudio de la Organización Marítima Internacional sobre Gases Invernadero (OMI, 2014) determina que el transporte marítimo emite el 2.2\% de las emisiones de dióxido de carbón generadas por las actividades humanas. Puntualmente, el transporte marítimo internacional es responsable del 2.7\% de emisiones globales de CO2, del 5 al 12\% de SOx y del 17 al 31\% de NOx. El estudio de la OMI también hace una proyección de las emisiones de $\mathrm{CO} 2$, determinando que tales emisiones pueden llegar a incrementar entre un $50 \%$ al $250 \%$ para el año 2050.

De la misma forma, Colbertt (2007) establece que las emisiones de los buques contribuyen al aumento de la tasa de mortalidad por enfermedades cardiovasculares y cáncer pulmonar. El transporte marítimo estuvo relacionado con aproximadamente 60,000 muertes en todo el mundo, durante el año 2002. Las regiones más afectadas fueron Europa y Asia, donde coinciden una alta densidad poblacional en las costas con unos elevados niveles de contaminación ambiental procedente de los buques. Esta situación es más evidente en Asia del Este y Asia del Sur, donde se presenta una alta tasa de mortalidad por estas enfermedades y una elevada actividad portuaria y marítima.

Por lo tanto, la OMI (2014) señala que es necesario implementar medidas para reducir las emisiones de GEI y contaminación atmosférica procedente de los buques.

\section{La relación entre los objetivos de desarrollo sostenibles y el transporte marítimo}

En 2015, la ONU (2015) adoptó una nueva agenda para el desarrollo mediante la iniciativa "Transformar nuestro mundo: la agenda de 2030 para el desarrollo sostenible” y sus 17 Objetivos de Desarrollo Sostenibles (ODS) asociados. 
La Agenda 2030 establece que los países desarrollados y los países en desarrollo deben ejecutar las medidas necesarias para alcanzar la erradicación de la pobreza, la preservación del planeta, la creación de un crecimiento económico sostenido, inclusivo y sostenible, así como el fomento de la inclusión social.

La Agenda 2030 para el desarrollo sostenible destaca el papel del comercio internacional y, por consecuencia, del transporte marítimo como motor del crecimiento y desarrollo sostenible. Es importante destacar que el desarrollo sostenible debe tener un equilibrio en las siguientes tres dimensiones: la economía, el medio ambiente y la social. A pesar de lo anterior, ningún ODS está enfocado en el transporte marítimo.

Por su parte, la Asociación de Navieros Noruegos (2017) señala que el transporte marítimo tiene impacto en las tres áreas del desarrollo sostenible. En primer lugar, el transporte marítimo guarda relación con la dimensión del medio ambiente por las emisiones de gases y descargas por parte de los buques. En segundo lugar, el transporte marítimo está vinculado con la dimensión económica, ya que se debe asegurar el ambiente de trabajo para la gente del mar y garantizar que los servicios de transporte apoyen el crecimiento económico. Por último, el transporte marítimo está conectado con la dimensión social, porque este medio de transporte tiene impacto en la salud y seguridad pública, así como con el acceso a productos alimenticios.

En concreto, la Asociación de Navieros Noruegos (2017) clasifica la relación entre los ODS y el transporte marítimo en alto, mediano y bajo impacto, tomando en cuenta la contribución de este último para alcanzar las metas de los ODS.

Tabla 1. Clasificación de la Asociación de Navieros Noruegos para medir el impacto del transporte marítimo en las metas de los ODS

\begin{tabular}{|l|l|l|}
\hline \multicolumn{1}{|c|}{ Alto impacto } & \multicolumn{1}{c|}{ Medio impacto } & \multicolumn{1}{c|}{ Bajo impacto } \\
\hline El ODS 8: Trabajo Decente y & El ODS 1: Fin de la Pobreza; el & El ODS 10: Reducción \\
Crecimiento Económico; el & ODS 2: Hambre Cero, el ODS & de las Desigualdades; \\
ODS 9: Industria, Innovación & 3: Salud y Bienestar; el ODS 4: & el ODS 11: Ciudades \\
e Infraestructura; el ODS 13: & Educación de Calidad; el ODS 5: & y Comunidades Sos- \\
Acción por el Clima; el ODS 14: & Igualdad de Género; el ODS 6: & tenibles; y el ODS 15: \\
Vida Submarina; el ODS 16: & Agua Limpia y Saneamiento; el & Vida de Ecosistemas \\
Paz, Justicia e Instituciones & ODS 7: Energía Asequible y no & Terrestres. \\
Sólidas; y el ODS 17: Alianzas & Contaminante; y el ODS 12: Pro- & \\
para Lograr los Objetivos. & tección y Consumo Responsable. & \\
\hline
\end{tabular}

Nota: recuperado de Norwegian Shipowners' Association. (2017). Sustainable Development Goals: Exploring Maritime Opportunities. 
De la misma forma, la Asociación de Navieros Daneses (2018) también reconocen el apoyo del transporte marítimo para alcanzar las metas de los ODS. El sector de transporte marítimo danés acepta la clasificación realizada por la Asociación de Navieros Noruegos. Pero, los navieros daneses señalan que su gobierno y la comunidad internacional deben enfocarse en la realización de acciones para conseguir las metas de cuatro ODS relacionados con el transporte marítimo. Estos ODS prioritarios son: el ODS 8: Trabajo Decente y Crecimiento Económico, el ODS 13: Acción por el Clima, el ODS 14: Vida Submarina y el ODS 16: Paz, Justicia e Instituciones Sólidas.

Al lado de estas dos asociaciones navieras, la OMI (2018) menciona que, a pesar de que ninguno de los ODS está particularmente dedicado al transporte marítimo, este medio de transporte es clave de forma directa 0 indirecta para el logro de 11 metas, repartidas en 8 ODS, a través de la implementación de las disposiciones emanadas de sus acuerdos o normas internacionales. Es decir, los acuerdos internacionales de la OMI coadyuvan en el éxito de las metas de los ODS.

\section{La contribución de los convenios internacionales de la OMI para alcanzar las metas de los ODS 3 salud y bienestar y ODS 13 acción por el clima}

La comunidad internacional ha generado instrumentos legales internacionales para alcanzar las metas del ODS 3: Salud y Bienestar y el ODS 13: Acción por el Clima.

El marco legal internacional sobre el cambio climático tiene como instrumento base la Convención Marco de Naciones Unidas sobre Cambio Climático (CMNUCC), adoptado en Rio de Janeiro en el año 1992. Dicho convenio tiene como finalidad lograr la estabilización de las concentraciones de GEI en la atmósfera a un nivel que impida interferencias antropógenas peligrosas en el sistema climático.

La CMNUCC establece que los países suscribientes podrán aprobar decisiones a través de las Conferencia de las Partes con la finalidad de garantizar los objetivos y la aplicación efectiva de las disposiciones de esta. La Conferencia de las Partes es el órgano supremo de la convención, y desde 1995se realiza una vez al año. 
En 2015, la vigesimoprimera Conferencia de las Partes de la CMNUCC fue realizada en París (COP21). En el marco de la COP21, las Partes de la Convención aprobaron el Acuerdo sobre Cambio Climático de París (el Acuerdo de París.). El Acuerdo de París tiene como objetivo principal mantener el aumento de la temperatura media mundial muy por debajo de $2^{\circ} \mathrm{C}$ con respecto a los niveles preindustriales, y proseguir los esfuerzos para limitar ese aumento de la temperatura a $1,5^{\circ} \mathrm{C}$ con respecto a los niveles preindustriales. Con la finalidad de alcanzar el objetivo antes mencionado, las Partes se comprometieron a reducir las emisiones nacionales de GEI. Sin embargo, las emisiones de GEI procedentes del transporte marítimo internacional no están contempladas en las disposiciones del Acuerdo de París.

La OMI es la agencia de la Organización de las Naciones Unidas encargada de establecer normas para la seguridad y la protección marítima, así como la prevención de la contaminación ambiental procedente del transporte marítimo.

El plan estratégico de la OMI (2017) señala que sus acuerdos y normas internacionales abarcan tres temas específicos y un tema transversal. Los temas específicos son: seguridad marítima, protección marítima y protección del medio ambiente. El tema transversal es la facilitación del tráfico marítimo internacional. Es decir, las medidas destinadas a promover un transporte marítimo seguro, protegido y ecológicamente racional no deben afectar la eficiencia del transporte marítimo.

En consecuencia, los acuerdos de la OMI están relacionados en mayor o menor medida con algunas de las metas de los ODS. Particularmente, el Convenio Internacional para Prevenir la Contaminación por Buques es un claro ejemplo de esta situación (MARPOL). La OMI (2014) establece que el Convenio MARPOL contribuye a lograr las metas de los ODS 3 y ODS 13. El Convenio MARPOL y sus anexos buscan prevenir la contaminación del medio marino producido por los buques, derivada de su funcionamiento o por un accidente. Concretamente, el Anexo VI del Convenio MARPOL busca prevenir la contaminación atmosférica proveniente de las emisiones de los buques.

El ODS 13: Acción por el Clima, tiene como objetivo adoptar medidas urgentes para combatir el cambio climático y sus efectos. Específicamente, la meta 13.2 de los ODS tiene como finalidad incorporar medidas relativas al cambio climático en las políticas, estrategias y planes nacionales.

De la misma forma, el ODS 3: Salud y Bienestar, tiene como finalidad garantizar una vida sana y promover el bienestar en todas las edades. La 
meta 3.9 tiene como objetivo para 2030, reducir sustancialmente el número de muertes y enfermedades producidas por productos químicos peligrosos y la contaminación del aire, el agua y el suelo.

Luego, las disposiciones del Anexo VI del Convenio MARPOL buscan reducir la contaminación atmosférica provocada por los buques. Por tal motivo, tales disposiciones coadyuvan en el éxito de las metas antes mencionadas.

El Convenio MARPOL fue adoptado en 1973. Posteriormente, los miembros de la OMI han adoptado dos protocolos y varios códigos técnicos derivados del Convenio MARPOL.

El Convenio MARPOL tiene los siguientes anexos: Anexo I: Reglas para prevenir la contaminación por hidrocarburos, Anexo II: Reglas para prevenir la contaminación por sustancias nocivas líquidas transportadas a granel, Anexo III: Reglas para prevenir la contaminación por sustancias perjudiciales transportadas por mar en bultos, Anexo IV: Reglas para prevenir la contaminación por las aguas sucias de los buques, Anexo V: Reglas para prevenir la contaminación por las basuras de los buques, y Anexo VI: Reglas para prevenir la contaminación atmosférica ocasionada por los buques.

Particularmente, el Anexo VI del Convenio de MARPOL está directamente relacionado con las metas y acciones de los ODS 3: Salud y Bienestar y ODS 13: Acción por el Clima.

Al respecto, podemos señalar que las principales disposiciones del Anexo VI del Convenio MARPOL son las siguientes:

- Establecer límites mundiales de emisión de SOx y NOx generadas por los buques.

- Prohibir la emisión deliberada de sustancias que agotan la capa de ozono.

- Permitir el establecimiento de zonas de control de las emisiones donde los estándares son más estrictos en relación con las emisiones de SOx y NOx ocasionadas por los buques y las plataformas.

- Acotar las emisiones producidas durante el mantenimiento, servicio, reparación o disposición de equipos de refrigeración.

- Fijar medidas técnicas y operacionales obligatorias de eficiencia energética para reducir las emisiones de gases de efecto invernadero producidas por los buques. 
En el caso de los límites de emisiones de SOx, debemos destacar que hasta el 31 de diciembre de 2019 el límite mundial de emisión de SOx procedente de buques fue de $3.5 \%$ masa/masa. Actualmente, el límite mundial de emisiones de azufre es de $\mathbf{0 . 5 0 \%}$ masa/masa. Por su parte, el límite de emisión de azufre procedente de buques es de $\mathbf{0 . 1 0} \%$ masa/masa en las Zonas de Control de Emisiones (ECA). Actualmente las zonas del control de emisiones de SOx son: Mar Báltico, Mar del Norte, Norteamérica y Mar Caribe de los Estados Unidos.

El Consejo Marítimo Internacional y del Báltico (BIMCO, 2019) señala que el Anexo VI del Convenio MARPOL ha sido ratificado por 93 países, quienes concentran el $96.70 \%$ del tonelaje mundial de carga.

La comunidad internacional es consciente de la importancia de adoptar medidas para reducir las emisiones contaminantes procedentes de los buques. Por tal motivo, algunos tratados de libre comercio han comenzado a incluir la obligación de ratificar e implementar los instrumentos legales internacionales para prevenir la contaminación generada por el transporte marítimo internacional. Un ejemplo de lo antes expuesto es el Tratado de Asociación Transpacífico (TPP)

Al respecto, la Secretaría de Economía de México (s.f.), señala que el TPP es un acuerdo de libre comercio que tiene los siguientes objetivos: regular el acceso a los mercados, las reglas de origen de la mercancía, las actividades de las empresas estatales, el comercio electrónico y los servicios, entre otras actividades comerciales; proteger la inversión extranjera y la propiedad intelectual; así como facilitar las actividades de exportación de las Pequeñas y Medianas Empresas. El TPP está integrado por: Australia, Brunéi Darussalam, Canadá, Chile, Japón, Malasia, México, Nueva Zelanda, Perú, Singapur y Vietnam.

Adicionalmente, el TPP establece obligaciones más allá de la regulación de actividades comerciales. El Capítulo 20 titulado Medio Ambiente del TPP determina obligaciones en materia ambiental. Al respecto, el artículo 20.6: Protección del Medio Marino de la Contaminación por Buques, determina que las Partes contratantes deben tomar medidas para prevenir la contaminación del medio marino por buques. Igualmente, dicho precepto legal establece que las Partes deben cooperar entre sí para reducir las emisiones de GEI y de azufre procedente de contaminantes de los buques. A pesar de lo anterior, el TPP no contempla expresamente la obligación de adoptar las disposiciones del Anexo VI del Convenio MARPOL por parte de las Países suscribientes. La mayoría de los países parte del TPP han suscrito el Anexo VI del Convenio 
MARPOL. En contraste, Brunéi Darussalam, México y Nueva Zelanda no han ratificado el Anexo VI del Convenio MARPOL.

\section{La falta de implementación de las medidas del anexo VI del convenio por parte de México}

En 1992, México ratificó el Convenio MARPOL 1973 y su protocolo de 1978. Adicionalmente, México ha ratificado el Anexo I: Reglas para prevenir la contaminación por hidrocarburos, el Anexo II: Reglas para prevenir la contaminación por sustancias nocivas líquidas transportadas a granel y el Anexo V: Reglas para prevenir la contaminación por las basuras de los buques, del Convenio MARPOL. A pesar de que el Anexo VI del MARPOL entró en vigor en el año 2005, México no realizó acciones para adherirse al mismo durante sus primeros años de vida. Por tanto, el Senado de la República exhortó al presidente de la República a informar sobre las acciones para adherirse al Anexo VI en el año 2010

En 2012, el presidente en turno, Enrique Peña Nieto inició las acciones tendientes para adherirse al Anexo VI.

En 2016, Estados Unidos, Canadá y México crearon la Alianza del Clima, Energía Limpia y Medio Ambiente de América del Norte con la finalidad de reducir las emisiones provenientes del transporte marítimo en las zonas marinas de los tres países. La alianza tiene como objetivo inicial reducir las emisiones de gases de efecto invernadero del transporte marítimo y cooperar con los trabajos de la OMI a través de la implementación de la Zona de Control de Emisiones de América del Norte, integrada por los tres países. Al respecto, el Gobierno de los Estados Unidos (2016) señala que la alianza busca ampliar la oferta de energías limpias para el transporte marítimo internacional en América del Norte a través de iniciativas, políticas públicas nacionales y reformar a las leyes internas de los tres países miembro.

Sin embargo, México no ha podido integrarse a la ECA de América del Norte debido a que no ha implementado las medidas necesarias para que los buques reduzcan sus emisiones de azufre a $\mathbf{0 . 1 0} \% \mathrm{masa} / \mathrm{masa}$ cuando operen dentro de las zonas marítimas mexicanas, ni ha ofertado combustóleo con dichos límites de azufre dentro de sus puertos. Por lo tanto, solamente Canadá y Estados Unidos integran la zona de control de emisiones de América del Norte. 
Ese mismo año, México suscribió la adhesión al Anexo VI del Convenio MARPOL en el marco de la COP21 en París. Al respecto, SEMARNAT (2016) señala que el principal objetivo de la adhesión al Anexo VI del Convenio MARPOL es transitar a una economía baja en carbón.

A pesar de la firma del Anexo VI del Convenio MARPOL, tanto el entonces presidente Enrique Peña Nieto, como el actual presidente Andres Manuel López Obrador, han omitido el envío del Anexo VI del Convenio MARPOL al Senado de la República para su ratificación.

El Senado de la República ha solicitado al titular del Poder Ejecutivo Federal enviar a esa Cámara el Anexo VI del Convenio MARPOL para proceder con el análisis y, en su caso, a la aprobación. No obstante, el Poder Ejecutivo Federal no ha enviado al Senado de la República el Anexo VI del Convenio MARPOL para su ratificación debido a que México tiene barreras económicas y tecnológicas para implementar sus disposiciones. Un ejemplo de lo antes mencionado es que México no tiene la infraestructura necesaria para generar combustóleo con límites de azufre $0.50 \%$ masa/masa.

El gobierno mexicano es consciente de los beneficios económicosy de bienestar a la salud derivados del establecimiento de la ECA y de la implementación de las medidas del Anexo VI del Convenio MARPOL. Al respecto, la Secretaria de Medio Ambiente y Recursos Naturales (2012) señala que la implementación de la reducción de las emisiones de contaminación procedentes de buques, permitirá prevenir enfermedades respiratorias, fallecimientos prematuros y ausentismo laboral; obtener un ahorro económico entre 41 y 61 mil millones de dólares para el sistema de salud mexicano; crear oportunidades de negocio relacionadas con el mercado de combustible marítimo ecológico; y contribuir a las acciones para evitar el cambio climático. Después de todo, México no tiene la capacidad económica ni material para poner en marcha los estándares establecidos en las Zonas de Control de Emisiones.

En este sentido, la Secretaría de Energía (2018) señala que el sistema nacional de refinería no tiene la capacidad para generar combustóleo de ultra bajo contenido de azufre. Por tal motivo, dicha secretaría pronostica que la demanda de diésel aumentará en los siguientes años para cumplir con la emisión máxima de azufre de $\mathbf{0 . 5 0 \%}$ masa/masa con base en el Anexo VI del Convenio MARPOL. 


\section{Conclusiones}

El transporte marítimo es pieza fundamental para el crecimiento económico global. De igual importancia, las emisiones contaminantes procedentes de los buques tienen un impacto directo en la salud humana y la protección del medio ambiental.

Las metas de los ODS no contemplan directamente al transporte marítimo a pesar de su impacto en la economía y el medio ambiente. Asimismo, algunos instrumentos internacionales en la materia no contemplan disposiciones para fomentar la disminución de las emisiones de contaminantes atmosféricos ni de GEI procedentes del transporte marítimo como la Convención Marco de Naciones Unidas sobre Cambio Climático y el Acuerdo de París.

En cambio, la OMI ha generado varios instrumentos legales internacionales que pueden ayudar a alcanzar algunas metas del ODS 3: Salud y Bienestar y del ODS 13: Acción por el Clima. En concreto, la implementación de las disposiciones del Anexo VI del Convenio MARPOL permiten coadyuvar en el éxito de algunas metas de los ODS antes señalados.

El Anexo VI del Convenio MARPOL es un instrumento legal internacional vinculante que impone medidas para reducir las emisiones de GEI, NOx y SOx procedentes del transporte marítimo. A pesar de lo anterior, algunos países todavía no han puesto en marcha las medidas del Anexo VI del Convenio MARPOL. Como señala SEMARNAT (2012), la creación de zonas de control de emisiones y la adopción de las medidas del Anexo VI del Convenio MARPOL, generan beneficios para la salud de la población costera y ahorros al erario público. A pesar de lo antes expuesto, México no ha adoptado las medidas necesarias para integrarse a la ECA de América del Norte.

De la misma manera, los tratados comerciales internacionales comienzan a incluir medidas para prevenir la contaminación del medio ambiente marítimo, como ocurre con el TPP. No obstante, el TPP no obliga a las Partes contratantes a implementar las medidas del Anexo VI del Convenio de MARPOL.

En el caso concreto de estudio, México no ha ratificado dicho anexo ni puesto en marcha las medidas necesarias para adherirse a la ECA de Norteamérica debido a la falta de recursos económicos e infraestructura. 
Los tratados comerciales multilaterales o bilaterales pueden ser una herramienta eficaz para incentivar entre los países la ratificación e implementación de las medidas del Anexo VI del Convenio MARPOL. Por tal motivo, los tratados de comercio internacional deben incluir expresamente la obligación de ejecutar las medidas de protección ambiental contempladas en el Anexo VI del Convenio MARPOL.

\section{Referencias}

Aalbu, K., Gjølberg, M., Longva, T. (Core team). Norwegian Shipowners' Association. (2017). Sustainable Development Goals: Exploring Maritime Opportunities. Recuperado de: https://rederi.no/globalassets/dokumenter-en/all/fagomrader/ smi/dnv-gl-sdg-maritime-report.pdf

BIMCO (2019). BIMCO BUNKER TERMS 2018. Recuperado de: https://www.bimco.org/ contracts-and-clauses/bimco-contracts/bimco-bunker-terms-2018\#

Corbett, J. (noviembre 7 de 2007). Mortality from Ship Emissions: A Global Assessment. Recuperado de: https://pubs.acs.org/doi/full/10.1021/es071686z

Danish Shipping (junio de 2018). Danish Shipping and the Sustainable Development Goals. Recuperado de: https://www.danishshipping.dk/politik/csr/verdensmal/ download/Basic_Model_Linkarea_Link/1022/policy-paper_danish-shipping-andthe-sustainable-development-goals-june-2018.pdf

International Maritime Organization. (2015). Third IMO GHG Study 2014. Recuperado de: https://www.imo.org/en/OurWork/Environment/Pages/Greenhouse-GasStudies-2014.aspx

International Maritime Organization. (2017). Application of the Strategic Plan of the Organization. Recuperado de:

https://www.imo.org/en/About/Strategy/Pages/Default.aspx

International Maritime Organization. (s.f.) IMO and the Sustainable Development Goals. Recuperado de: http://www.imo.org/en/MediaCentre/HotTopics/Pages/ SustainableDevelopmentGoals.aspx

International Maritime Organization. (2018). Resolution MEPC. 304(72), Initial IMO Strategy on Reduction of GHG Emissions from Ships. (abril de 2018) Recuperado de: https://wwwcdn.imo.org/localresources/en/KnowledgeCentre/ IndexofIMOResolutions/MEPCDocuments/MEPC.304(72).pdf

International Maritime Organization. (2020). IMO 2020 A breath of Fresh Air. Recuperado de: https://imo-newsroom.prgloo.com/resources/imo-2020-a-breath-of-fresh-air

Londres, Organización Marítima Internacional (OMI) (17 de febrero de 1978). Anexo VI: "Reglas para prevenir la contaminación Atmosférica ocasionada por los Buques", Regla 14 del 19 de mayo de 2005.

Martner Peyrelongue, C., Pérez Sánchez, A. Instituto Mexicano del Transporte. (2020). Evolución de la carga por los puertos mexicanos: Impactos diferenciados del COVID-19 por tipo de carga y litoral. Segunda Parte. Recuperado de: https://imt. $\mathrm{mx}$ /images/files/GRAL/documentos/GacetaAMANAC-IMT.pdf 
Naciones Unidas, Convención Marco de las Naciones Unidas sobre el Cambio Climático (1992).

Naciones Unidas. (2015). Transformar nuestro mundo: la Agenda 2030 para el Desarrollo Sostenible. Recuperado de: https://unctad.org/meetings/es/SessionalDocuments/ ares70d1_es.pdf

Naciones Unidas, Convención Marco de las Naciones Unidas sobre el Cambio Climático, "Acuerdo de París", 12 de diciembre de 2015.

Notteboom, T., Pallis, T. (2020). IAPH-WPSP Port Economic Impact Barometer. Recuperado de: https://sustainableworldports.org/wp-content/uploads/2020-0608-COVID19-Barometer-Report.pdf

Secretaría de Economía. (2015). Resumen Ejecutivo del Acuerdo de Asociación TransPacífico. Recuperado de: https://www.gob.mx/cms/uploads/attachment/file/23615/ TPP_resumen_ejecutivo.pdf

Secretaría de Energía. (2018). Diagnóstico de la Industria de Petrolíferos en México. Recuperado de: https://www.gob.mx/cms/uploads/attachment/file/416899/ Parte_1_vf.pdf

Secretaría de Medio Ambiente y Recursos Naturales. (2012). Proyecto para la Evaluación de Impactos y Beneficios del Anexo VI de MARPOL y la ECA para México. Recuperado de: https://19january2017snapshot.epa.gov/sites/production/files/2014-05/documents/2impactsbenefits.pdf

Secretaría de Medio Ambiente y Recursos Naturales. (2016). México en la COP21, listo y decidido a enfrentar el cambio climático. Recuperado de: https://www.gob.mx/ semarnat/prensa/mexico-en-la-cop21-listo-y-decidido-a-enfrentar-el-cambioclimatico

Senado de la República. (2010). Dictamen de la Comisión de Medio Ambiente Recursos Naturales y Pesca, a la Propuesta con Punto de Acuerdo por el que se Solicita al Titular del Ejecutivo Federal un Informe relacionado con las Acciones Desarrolladas Tendientes a la adhesión del Anexo VI del Convenio Internacional MARPOL - Reglas para prevenir la Contaminación Atmosférica por los Buques. (octubre de 2010). Recuperado de: https://infosen.senado.gob.mx/sgsp/gaceta/61/2/2010-10-19-1/ assets/documentos/internacional_marpol.pdf

Senado de la República. (2017). Dictamen de la Comisión de Relaciones Exteriores Organismos Internacionales, a la Proposición con Punto de Acuerdo por el que se exhorta al Titular del Poder Ejecutivo Federal a Enviar al Senado para su Análisis

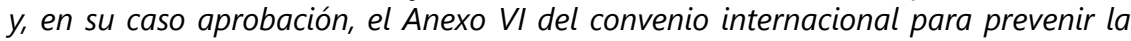
Contaminación por los Buques. (Septiembre de 2013). Recuperado de: https:// infosen.senado.gob.mx/sgsp/gaceta/63/3/2017-09-26-1/assets/documentos/ Dict_Rel_Ext_OI_MARPOL.pdf

The White House (2016). Leaders' Statement on a North American Climate, Clean Energy, and Environment Partnership. (junio 2016) Recuperado de https:// obamawhitehouse.archives.gov/the-press-office/2016/06/29/leaders-statementnorth-american-climate-clean-energy-and-environment

United Nations Conference on Trade and Development. (2020). Review Of Maritime Transport 2019. Recuperado de: https://unctad.org/en/PublicationsLibrary/ rmt2019_en.pdf 